\title{
Hair defect-photosensitivity-intellectual disability syndrome
}

INSERM

\section{Source}

INSERM. (1999). Orphanet: an online rare disease and orphan drug data base. Hair defect-photosensitivity-intellectual disability syndrome. ORPHA:1408

Calderon-Gonzalez-Cantu syndrome is characterized by the association of stubby, coarse, sparse and fragile hair, eyebrows and eyelashes with photosensitivity and nonprogressive intellectual deficit without a demonstrable metabolic aberration. 